\title{
An Improved Gray Multi-focus Image Fusion Algorithm Based on Dual-Tree Complex Wavelet Transform
}

\author{
Wang Jianyu \\ School of Transportation \\ Huanggang Normal University \\ Hubei Huanggang, China \\ whutliuxiaojun@126.com
}

\author{
Liu Xiaojun* \\ School of Electronic \& Information \\ Huanggang Normal University \\ Hubei Huanggang, China \\ whutliuxiaojun@126.com
}

\begin{abstract}
In this paper, a gray multi-focus image fusion algorithm based on fuzzy theory classification and DualTree Complex Wavelet Transform is proposed in accordance with fusion of gray multi-focus images. Fuzzy theory is introduced to remove uncertainty when choosing sub-band coefficients in smooth regions after Dual-Tree Complex Wavelet Transform. Fuzzy membership is used to measure the mapping relation between different feature types of images and corresponding pixels gray values. The uncertainty probably exists in fusion process is removed efficiently. Efficient fusion and detail enhancement of images are realized. Simulation results verify the effectiveness and superiority of proposed algorithms.
\end{abstract}

Keywords- Multi-focus Image Fusion ; Fuzzy Theory ; Dual-Tree Complex Wavelet Transform

\section{INTRODUCTION}

Currently, there are mainly two multi-focus image fusion (Multi-focus Image Fusion) methods: one is based on pyramid transform and the other is based on wavelet transform ${ }^{[1]}$. Owing to the djacent decomposition layers data of pyramid transform is redundant, and no direction ${ }^{[2]}$, while the wavelet transform has a good time - frequency localization properties and non-redundancy and nondirectional, therefore wavelet transform as a signal and image processing MRA tools, discrete wavelet transform (DWT) is widely used. However, there are serious flaws in the translation invariance and direction selectivity leads to wavelet coefficients larger changes in energy distribution, and the output image information containing a large redundancy, so increased computational traditional DWT. Despite complex wavelet transform (CWT) can overcome these problems, but due to CWT input form is plural, complex wavelet tree structure complete reconstruction filter is very difficult.

N.G.Kingsbury has proposed dual tree complex wavelet transform (DT-CWT) ${ }^{[3-4]}$, which is a method of CWT fully reconstructed, during the DT-CWT process, the source image to generate a small amount of the offset signal, each decomposition scale factor does not change large, and DT-CWT has good directional selectivity coefficient can be separated from six different directions, the source image can be decomposed more details, minutiae sharper edges characterize the image.

\section{APPLICATION AND REASONING TACTICS OF FUZZY THEORY IMAGES}

In recent years, more and more image processing studies are used to Fuzzy Theory. Due to there is uncertainty of image data during the image data fusion, to achieve an accurate understanding of image fusion, fuzzy theory is accurate tool for dealing with variables ${ }^{[5-6]}$.

\section{A. Theory Images}

The fundamental principles of fuzzy theory is used to image processing is the mapping relationship between different types of features fuzzy membership metric image with the corresponding pixel gray values, the main process is:

1. Delimited the image pixels and extracted features from the image, commonly used for the gray values, texture characteristics and so on.

2. Assigned this membership function of the feature type to each pixel, it represents the pixel point $x$ of image $\mathrm{j}$ belongs to the membership of type $i$. After, according to the concept of fuzzy theory to convert the pixel and type [0,1],Their size reflects the degree of membership of $\mathrm{x}$ of type $\mathrm{i}$, the closer to 1 , the higher the degree of $\mathrm{i} x$ belongs.

3. Define the possibility measure used to indicate the mixing degree of membership function between the two, and determine the presence of various types of images.

4. Fused all the possibility measure associated with type i to obtain global possibility of type i.

5. Reasoning strategy designed to make the type i guess.

\section{B. Based on "if-then" reasoning strategies}

"If-then" is a conditional statement that contains fuzzy logic, In its simplest form "if-then" strategy is: If $\mathrm{x}$ is A then $\mathrm{y}$ is $\mathrm{B}$, it means that if $\mathrm{x}$ is $\mathrm{A}$, then $\mathrm{y}$ is $\mathrm{B}, \mathrm{A}$ and $\mathrm{B}$ are fuzzy linguistic variables, the relationship between $A$ and $\mathrm{B}$ is the fuzzy relationship, denoted as $\mathrm{A} \rightarrow \mathrm{B}$, " $\mathrm{X}$ is $\mathrm{A}$ " is a prerequisite, "y is B" is the conclusion ${ }^{[7-8]}$.

Process applications "if-then" reasoning strategies can be divided into the following three steps: 
Discuss all possible preconditions.

Determine "if-then" rules of membership of each preconditions.

By each preconditions of membership and conditions of possibility inference the extent of conclusion is true, that is the conclusion of the membership.

The process of fuzzy reasoning that is infer new conclusions from we have known various preconditions. This method for image fusion, it can effectively eliminate there may be uncertainty during integration.

\section{A GRAY MULTI-FOCUS IMAGE FUSION} ALGORITHM BASED ON FUZZY THEORY CLASSIFICATION AND DUAL-TREE COMPLEX WAVELET TRANSFORM

This paper presents an algorithm that based on Fuzzy Theory discrimination and gray multi-focus image fusion of DT-CWT by Combining with multi-focus image imaging mechanism and characteristics of the human visual system.

\section{A. Fusion framework}

Fig. 1 is based on multi-focus image fusion framework DT-CWT shown, first, the source image layer DT-CWT decomposition of $\mathrm{J}$, obtained $(6 \mathrm{~J}+1)$ subband images. Among them, Low-frequency component represents the contour information of the source image and highfrequency component represents the details of the source image. Select the appropriate fusion rules for the low frequency and high frequency components are fused, its dual tree complex wavelet inverse transform, you can get the fused image. Below two images for example, follow these steps:

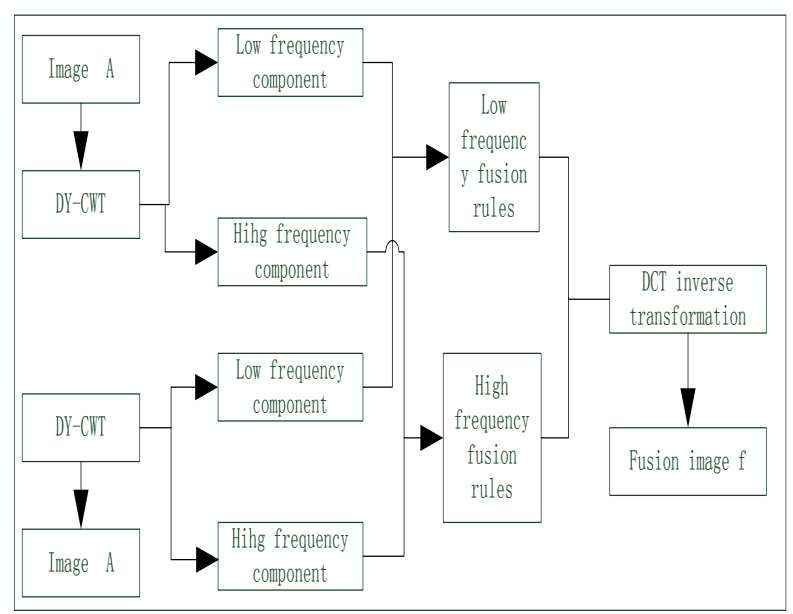

Figure 1. Multi-focus image fusion framework

\section{B. Fusion algorithm implementation process}

The choice of fusion rules is a critical step in Multiresolution image fusion analysis, and after the merger of different sub-band coefficients to obtain the best quality image fusion, the dotted line in Figure 1 is the next focus of the study. People have studied multiple three fusion rules include: the maximum value selection method (MS), each sub-band by selecting the largest coefficient judgment rules. Burt and Kolczynaki proposed by the weighted average method (WA). the method discussed correlation between the two sub-bands normalized in the area of the window

The above fusion rule ignores some useful information and sensitive to noise, method one coarse-scale decomposition, the image becomes smoother, making it impossible to accurately distinguish whether an image factor which should be selected as the focus factor. In the second method, the weights are calculated by a linear function, we can not describe the contribution uncertainty of each source image. method three, choose the operator to make fusion factor is entirely dependent on the larger area energy while ignoring other factors correlation coefficient.

This paper presents a new fusion rule based on combining Fisher discrimination with fuzzy theory discrimination. Six dimensions of the feature vector can be obtained through the DT-CWT sub-band can be used as training differentiator. As with the discriminator output decision diagram choose different dimensions, different decomposition layer sub-band coefficients of the two images. The smooth areas select sub-band coefficients exist some uncertainty in the two image, this situation has led to the presence of non-discriminating regions. $\mathrm{T} o$ address this uncertainty, we can use fuzzy theory, the useful information of each image as much as possible integration into the result image, to this end, we have proposed an algorithm based on fuzzy theory discrimination, considering the three fusion rules output. The dotted line in Figure 1 fusion rule is shown in Fig.2, Fig. 2 is an example of high frequency components.

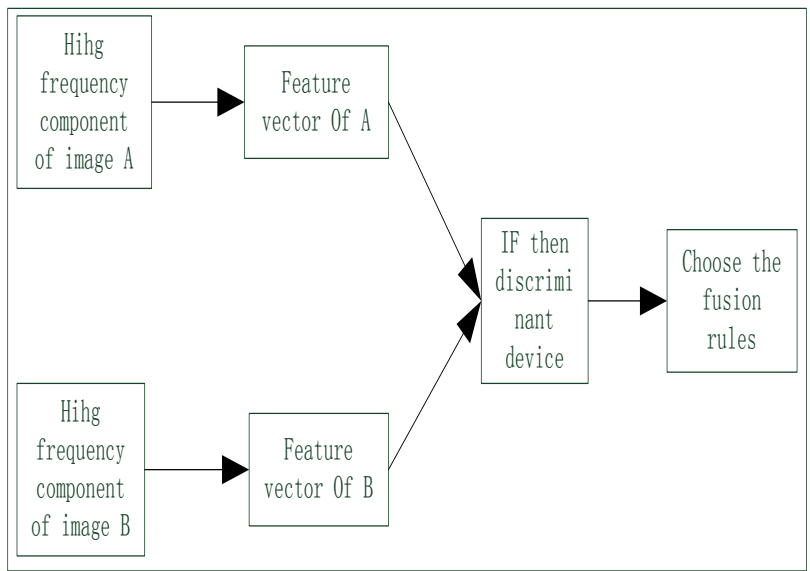

Figure 2. High-frequency coefficient fusion rules.

IV. SIMULATION AND ANALYSIS

Simulation experiments using two sizes are $256 \times 256$ multi-focus "clock" image. Two images before registration has been conducting fusion experiments. Shown in Figure 3, "clock" image focal point A on the left side, so the left side of the area more clearly, "clock" image focal point B on the right side, so the right side of the area more clearly.

To compare and verify the validity and correctness of this chapter fusion algorithm, experiments using the same scene of gray-scale of multi-focus image simulation fusion, with three kinds of more typical fusion algorithm comparison, the first one is based on Laplace pyramid transform (Laplacian Pyramid Transform, LPT) image fusion algorithm, decomposition level is 4 layer, and the top LPT sub-band coefficients are fused using the 
weighted average method. The second is based on Discrete Wavelet Transform( DWT) image fusion algorithm using "db4" wavelet filter. the third is based on the DT-CWT image fusion algorithm (DT-CWT Simple). Both decomposition based on MRI image fusion algorithm image decomposition stages are four. To enable comparability between the various fusion algorithms, both above fusion algorithms are used the low frequency coefficient part weighted average and use the greater highfrequency coefficients regional energy integration rules during fusion treatment. Figure 4 (a), (b) shows the gradation to be fused multi-focus source image,(c) (f) is the integration of the results of various algorithms.

We can draw subjective evaluation from the fusion experimental: fusion algorithm presented in this chapter not only effectively eliminate focuses differences in the

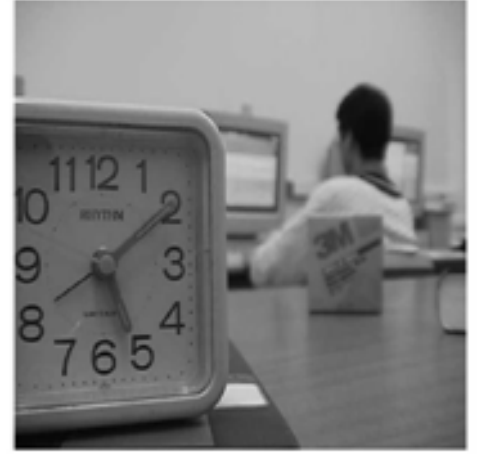

(a) source image $\mathcal{k}$ source image, but also improves the clarity of the image, get the best results in the fusion of four fusion algorithms.

In order to quantitatively evaluate the performance of different fusion algorithms in multi-focus image fusion. In this paper, entropy $(\mathrm{H})$, root mean square error (RMSE), mean gradient (G), spatial frequency (SF) as an objective evaluation criteria, evaluation of fusion as shown in Table $1^{[9-10]}$.

As can be seen from the fusion effect evaluation, this algorithms compare to other algorithm in simulation get the image entropy $(\mathrm{H})$,root mean square error (RMSE), mean gradient (G),spatial frequency (SF) have been greatly improved, this is mainly due to the use of different multi-resolution decomposition tool and different fusion rules caused.

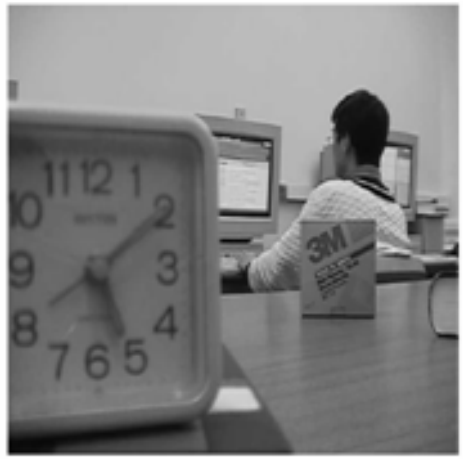

(b) source image $B$

Figure 3. Figure 3 to be fused images (a) source image A. (b) source image B

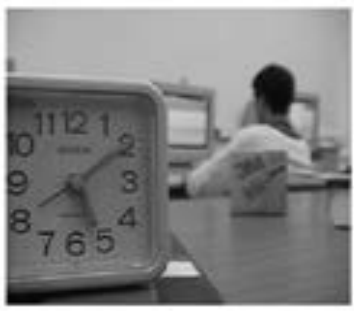

(a) left focus

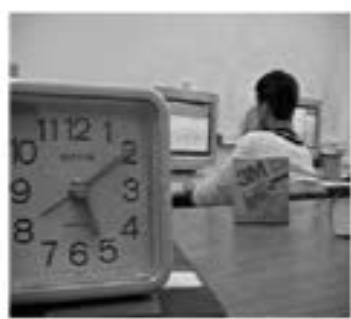

(d) D'WT :

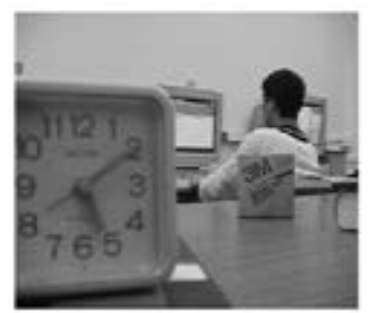

(b) right focus

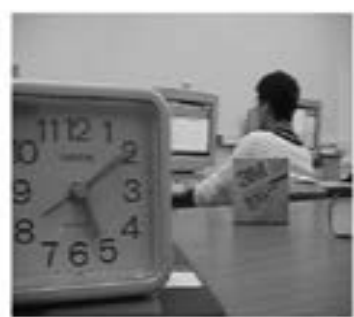

(e) DT-CH'T

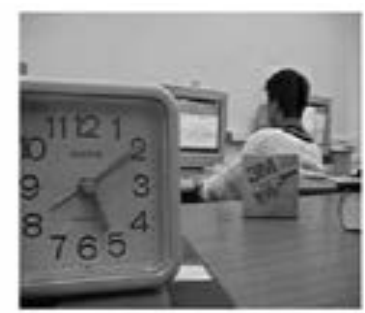

(c) fusion image of LPT

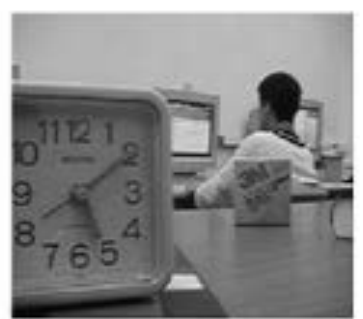

(f) this paper

Figure 4. gray multi-focus image fusion experiments.(a) left focus. (b) right focus. (c) fusion image of LPT. (d) fusion image of DWT. (e) fusion image of DT-CWT Simple (f) fusion image of proposed

TABLE I.

EVALUATION OF THE FUSION ALGORITHM

\begin{tabular}{|c|c|c|c|c|}
\hline \multirow{2}{*}{ Fusion method } & \multicolumn{4}{|c|}{ Evaluation indicators } \\
\cline { 2 - 5 } & $\mathrm{H}$ & RMSE & $\mathrm{G}$ & SF \\
\hline LPT & 8.0619 & 3.1268 & 12.9852 & 12.5278 \\
\hline DWT & 8.0873 & 1.9177 & 13.2902 & 12.9268 \\
\hline DT-CWT & 8.1049 & 1.0495 & 16.9504 & 13.2052 \\
\hline This Paper & 8.2598 & 0.7566 & 18.6542 & 13.4956 \\
\hline
\end{tabular}


V.

\section{CONCLUSION}

Based on the multi-resolution analysis of image fusion framework: multi-resolution analysis algorithm is the basis of image fusion, the fusion rule is the core of image fusion, the fusion effect evaluation is the key of image fusion. In view of this, a gray multi-focus image fusion algorithm based on fuzzy theory classification and Dual-Tree Complex Wavelet Transform is proposed in accordance with fusion of gray multi-focus images in this paper. Through the simulation experiment, the effectiveness of the algorithm is verified.

\section{ACKNOWLEDGEMENTS}

The project was supported by the Doctoral Scientific Fund Project of Huanggang Normal University (Grant No. 2013031103), and the Scientific Research Program of Educational Commission of Hubei Province of China (Grant No. 20142905).

\section{REFERENCES}

[1] Shi W, Zhu C, Tian Y, et al. Wavelet-based image fusion and quality assessment[J]. International Journal of Applied Earth Observation and Geo-information, 2005, 6(3):P241-251P.
[2] G. Piella, H. Heijmans, A new quality metric for image fusion[C]. Proceeding of IEEE International Conference on Image Processing, Barcelona, Spain, 2003. P173-176.

[3] Kingsbury N G. Image Processing with Complex Wavelet[J]. Phil. Trans. Royal Society London Ser. A357(1999), P2543-2560.

[4] Kingsbury N. The dual-tree complex wavelet transform: a new efficient tool for image restoration and enhancement[J]. Proc. EUSIPCO 98, Rhodes, Greece, P319-322.

[5] Teng Jionghua, Wang Suhuan, Zhang Jingzhou, et al. Proceedings - $20107^{\text {th }}$ International Conference on Fuzzy Systems and Knowledge Discovery, FSKD 2010, 2010, P546-550.

[6] P. J. Burt, R. J. Kolczynski. Enhanced image capture through fusion[C]. Proceedings of the 4th International Conference on Computer Vision, 1993, P173-182.

[7] Morita Satoru. Generating shaded image with lighting using image fusion space[J]. Lecture Notes in Computer Science, 2010, P584893.

[8] Sunil Kumar, Rajat Gupta, Nitin Khann. Text extraction and document image segmentation using matched Wavelets and MRF model[J]. IEEE Trans. On Image Processing, Vol.16, NO.8, 2007: P2117-2128.

[9] Hossny M., Nahavandi S., Creighton D. Image fusion performance metric based on mutual information and entropy driven quadtree decomposition[J]. Electronics Letters, 2010, 46(18):P1266-1268.

[10] Yang Y. Y, Chiam T.C. Rule discovery based on rough set theory. Proceedings of $3^{\text {rd }}$ International Conference on Information Fusion[J]. Electronics Letters, 2012, 38(7):P313- 315. 